\title{
How Well Can We Predict the Toxicity of Pesticide Mixtures to Aquatic Life?
}

\author{
Jason B Belden, ${ }^{*}+$ Robert J Gilliom, $\S$ and Michael J Lydy $\neq$ \\ tDepartment of Environmental Studies, Baylor University, Waco, Texas 76798, USA \\ §US Geological Survey, Sacramento, California 95819, USA \\ łFisheries and Illinois Aquaculture Center and Department of Zoology, Southern Illinois University, Carbondale, Illinois 62901, USA
}

(Received 6 September 2006; Accepted 11 February 2007)

\begin{abstract}
Results of published pesticide mixture toxicity experiments conducted with aquatic organisms were compiled and evaluated to assess the accuracy of predictive mixture models. Three types of models were evaluated: Concentration addition (CA), independent action (IA), and simple interaction (SI). The CA model was the most often tested (207 experiments), followed by SI (59) and IA (37). The reviewed experiments are listed in the Supplemental material to provide a resource for future investigators. The predictive accuracy of each model was quantified for each experiment by the model deviation ratio (MDR), which was calculated by dividing the predicted toxicity by the observed toxicity. Eighty-eight percent of all experiments that evaluated the CA model had observed effective concentrations within a factor of 2 of predicted values (MDR values from 0.5-2.0). The median MDR was 1, about $5 \%$ of MDRs were less than 0.5 , and about $5 \%$ were greater than 2, indicating unbiased estimates overall. The predictive accuracy of CA and IA models was influenced, however, by the different modes of action (MOA) of the pesticides. For experiments with pesticides with the same MOA, CA more accurately predicted effective concentrations for more experiments compared to IA, which tended to underpredict toxicity. The IA model was somewhat more accurate than the CA model for most mixtures with different MOAs, but in most cases there were relatively small differences between the models. Additionally, $80 \%$ of SI experiments had an MDR value below 2.0 despite a bias towards experiments that are likely to have an interaction. Thus, results indicate that the CA model may be used as a slightly conservative, but broadly applicable model with a relatively small likelihood of underestimating effects due to interactions.
\end{abstract}

Keywords: Mixture Pesticide Concentration addition Independent action Interaction

\section{INTRODUCTION}

Concerns over the potential of combined effects from cooccurring pesticides in aquatic systems have lead to numerous studies of pesticide mixtures during the past $30 \mathrm{y}$. Assessment of streams throughout the United States and Europe showed that pesticides frequently are detected and most often occur in mixtures (Gilliom et al. 1999; Chevre et al. 2006; Gilliom et al. 2006). Toxicity studies have examined the effects of mixtures of multiple pesticides following laboratory exposures (references listed throughout text and Supplemental material), evaluated mixtures at a mesocosm level (Hoagland et al. 1993; Fairchild et al. 1994; Carder and Hoagland 1998; Cuppen et al. 2002; Boone and James 2003), and evaluated pesticides as potential toxic components of more complex mixtures that include other types of contaminants (Norberg-King et al. 1991; Amweg et al. 2006). This study focuses on the effects of mixtures of pesticides, recognizing, however, that pesticides represent only 1 class of stressors that organisms may be exposed to in the environment.

Due to the large number of different pesticide mixtures that are present in the environment (Gilliom et al. 2006), it is not feasible to test toxicologically each potential mixture. Thus, robust predictive models are needed that can estimate the toxicity of mixtures with acceptable accuracy (Vighi et al. 2003; Lydy et al. 2004). Furthermore, in order for mixture models to be useful in ecological risk assessments,

* To whom correspondence may be addressed: jason_belden@baylor.edu. knowledge of the uncertainty and potential bias of the models is necessary. Although nearly all experiments reviewed for this study tested a predictive model and provided a measure of deviation from that model, many different techniques and approaches were used. To compare results among studies, predictive models must be grouped on the basis of general experimental design, and a uniform scale must be used to express deviations between predictions and observations.

The primary objective of this study was to evaluate the accuracy of toxicity models for predicting the toxicity of pesticide mixtures. Although the predictive accuracy of specific models was discussed in nearly every publication of mixture studies and in previous reviews (Deneer 2000; Lydy et al. 2004), the quantitative measures that have been used are inconsistent and difficult to compare among studies. In the present study, a uniform approach was used to evaluate the predictive accuracy of mixture models across experimental designs and terminologies. A 2nd objective was to provide a readily accessible compilation of published pesticide mixture experiments and the findings of individual experiments that evaluated mixture toxicity models. Although 2 previous reviews addressed pesticide mixtures in aquatic systems, Deneer (2000) addressed only concentration addition (CA) models, Lydy et al. (2004) focused primarily on the broad issues regarding the future regulation of pesticide mixtures, and neither developed an accessible database for specific pesticide mixtures and specific aquatic organisms. 


\section{METHODS: DEVELOPMENT OF A PESTICIDE MIXTURE DATABASE}

\section{Identification of experiments}

Manuscripts that describe experiments with pesticide mixtures were identified from previous reviews (Deneer 2000; Altenburger et al. 2003; Lydy et al. 2004), searches of bibliographic databases (such as Web of Science, Thomson Scientific and Healthcare, Stamford CT, USA; Agricola, US Department of Agriculture, Washington DC, USA; First Search/WorldCat, HW Wilson, Bronx, NY, USA), and through cited literature searches (Web of Science) of key manuscripts that have been cited heavily (Faust et al. 1993; Pape-Lindstrom and Lydy 1997; Altenburger et al. 2000; Backhaus et al. 2000; Faust et al. 2000). Only mixture experiments that were conducted with mixtures of active ingredients were included. Studies that evaluated active ingredients applied in pure form versus applied in formulations were not included in the database. Testing of formulations that contain adjuvants requires each individual adjuvant to be tested and included in the predictive model. Additionally, specific criteria were used to avoid biasing the database with similar experiments from a single study. For example, experiments conducted with a particular pesticide combination and species were included if they reflected independent experiments and were reported in different manuscripts, but duplicate experiments in the same manuscript were entered as a single entry. Second, within a manuscript, if multiple ratios of the same pesticides were evaluated (such as in isobole studies), only the mixture closest to equipotent, as calculated from the median lethal concentration, was considered. However, if different ratios were used to test different predictive models, both models were represented in the database. Similarly, if several concentrations of a compound were tested for potentiation, only the highest concentration was entered as a worst-case scenario. Selection of 1 ratio per experiment prevented biasing the database towards experiments that used multiple ratios, as the primary synthesis of the database was based on pesticide combinations and species tested and not on differences in results based on ratios. Third, if a single experiment was used to evaluate 2 predictive models, the data were entered twice, once for each model. Finally, the experiment had to be conducted in a way that a model deviation ratio (MDR) could be calculated.

\section{Information collected for each experiment}

Several key types of information were identified for each experiment, including pesticides involved, species tested, higher taxonomic classification, endpoint of the toxicity test, duration of the toxicity test, the model tested (CA, independent action [IA], or simple interaction [SI]), the MDR value, reports of significant deviation from model, and the reference of the manuscript. Broader quality-control considerations, such as the reliability of measured concentrations and the adequacy of experimental controls, were not included in the current evaluation.

A necessary step in assessing pesticide mixtures is defining specific classification schemes. Pesticides can be classified in a variety of ways. In the most general sense, they can be organized by the type of pest targeted, defined as pesticide use groups, such as insecticides and herbicides. In a more detailed framework, pesticides can be grouped into classes of compounds that have similar chemical structures and modes of action. The term mode of action (MOA) is defined as a series of key processes that begins with the interaction of a pesticide with a receptor site and proceeds through operational and anatomical changes in an organism that result in sublethal or lethal effects (USEPA 2000). For this study, both general (based on major pesticide use groups) and specific (based on MOA) classification schemes were used.

\section{Classification of predictive models}

Methods and terminology used in mixture studies have changed steadily during the time frame that the reviewed studies were conducted (1973 to 2006). Despite such changes, most of the studies could be placed within the general theoretical framework proposed by Hewlett (1969). Within this framework, toxicants may act independently or jointly and may or may not interact. On the basis of these categories, each experiment was placed in 1 of 3 groups, CA, IA, or SI, according to the type of predictive model that was used as a test.

The lst group of experiments is based on CA, the concept that when toxicants have a similar MOA, they act jointly in an additive manner after normalizing for potency (reviewed by Altenburger et al. 2003). Following normalization for potency, the concentrations of all toxicants can be summed to obtain a value that can be used to predict toxicity. According to the CA model, the total concentration of a mixture at which a certain effect is expected can be calculated using the following equation (Faust et al. 2000):

$$
E C x_{\text {mix }}=\left(\sum_{i=1}^{n} \frac{p_{i}}{E C x_{i}}\right)^{-1}
$$

where $E C x_{\text {mix }}$ is the total concentration of the mixture that causes $x$ effect; $p_{i}$ indicates the proportion of component $i$ in the mixture; $n$ indicates the number of components in the mixture, and $E C x_{i}$ indicates the concentration of component $i$ that would cause $x$ effect.

In practice, many experiments in the CA group have used a normalized toxicant concentration scale (toxic units [TU]) that allows addition of the concentrations of each toxicant. TUs can be calculated as follows:

$$
\operatorname{sum} T U=\sum_{i=1}^{n} \frac{\mathrm{C}_{i}}{E C x_{i}}
$$

where $C_{i}$ is the exposure concentration of the $i$ th chemical in the mixture and $E C_{x i}$ and the other variables are defined as stated for Equation 1.

The 2nd group of experiments is based on IA, the concept that toxicants that have dissimilar MOA act independently. Conceptually, this model is a statistical approach to predict the likelihood that 1 of multiple possible events will occur (reviewed by Altenburger et al. 2003). Accordingly, the effect of the total mixture concentration can be predicted by the expected effect of each component, using the following equation (Faust et al. 2000):

$$
E\left(c_{\text {mix }}\right)=1-\prod_{i=1}^{n}\left(1-E\left(c_{i}\right)\right)
$$

where $E\left(c_{\text {mix }}\right)$ is the total effect of the mixture and $E\left(c_{i}\right)$ is the effect expected from component $i$. Although special cases have been described with complete or no correlation of sensitivity within the population (resulting predictive toxicity ranging from the effect of the most toxic component to 
straight effect addition), this information rarely is known and thus is not useful in an a priori evaluation of toxicity.

The 3rd group of experiments is based on the concept of interaction. Each of these experiments was designed such that 1 of the toxicants was evaluated at a concentration well below that necessary to add to toxicity through either CA or IA models. Because only interaction toxicity is tested, the experiments are referred to as SI. An interaction is defined as 1 of the toxicants influencing the toxicity of the other toxicant through an indirect mechanism. The most studied examples of interaction in ecotoxicology are changes in toxicokinetics. For example, atrazine has been shown to increase the transformation rate of organophosphate insecticides; thus increasing toxicity (Belden and Lydy 2000, reviewed by Lydy et al. 2004), and organophosphate insecticides (OPs) have been shown to inactivate esterase, resulting in reduced detoxification of other OPs and pyrethroids insecticides, which results in greater toxicity than would be expected (Denton et al. 2003; Belden and Lydy 2006).

Although specific examples have been well studied, models to predict interactions are not available. In most cases, interactions are not tested for directly, but are discovered as deviations from CA or IA models. However, in some studies, an alternative study design is used in which one of the toxicants is applied at a concentration that is too low to appreciably influence overall toxicity according to either CA or IA models. For example, in a recent study, chlorpyrifos was evaluated as an interactive agent when applied with esfenvalerate at a concentration of chlorpyrifos that was below an ECl value (Belden and Lydy 2006). This type of design typically is employed to evaluate potential interactions, which sometimes is referred to as potentiation or synergism because most experiments tend to focus on higher-than-expected toxicity. Because interaction currently cannot be predicted, the assumed model is the concentration-response curve of the toxicant that is expected to cause toxicity (Belden and Lydy 2006).

\section{Size and breadth of database}

Forty-five published manuscripts detailing 303 experiments were identified that tested predictive mixture models and contained sufficient data for calculating MDR values. Summary data from these experiments are compiled in the Supplemental Material section to provide a resource for investigators seeking empirical results for specific mixtures. From the identified experiments, 207 were classified as CA (Supplemental Tables 1-3), 37 as IA (Supplemental Table 4), and 59 as SI (Supplemental Table 5). References for these manuscripts are listed in the Supplemental Tables. The number of experiments retained in the database is similar to the number reviewed by Deneer (2000), who identified 26 studies involving 202 mixture experiments all reportedly based on CA. The current study included 37 studies involving 207 mixture experiments for CA. The reduction in experiments relative to overall studies results from the different acceptance criteria that were used.

Further delineation of experiments indicated that most studies tested pesticides from the same pesticide use group (i.e., all herbicides or all insecticides) with 113 and 17 experiments conducted for CA and IA, respectively (Supplemental Tables 1 and 4). Mixtures of pesticides from different use groups (i.e., an herbicide with an insecticide) ranked 2 nd in the number of experiments conducted, with 64 and 13 experiments conducted for CA and IA, respectively (Supplemental Tables 2 and 4). The least frequent scenario was the testing of compound mixtures with the same MOA, with 30 and 7 experiments conducted for CA and IA, respectively (Supplemental Tables 3 and 4). Studies testing SI were not separated based on MOA, because the basis for the interaction is generally through a secondary affect on the organism.

For nearly all studies conducted using CA and SI models, MDR values could be calculated because the studies were based on effective concentrations and changes in effective concentrations (see later description of MDR). However, over 50 other studies evaluating mixtures using IA or simplified effect addition models only evaluated shifts in effect (e.g., Macek 1975; Hayes et al. 2006), which did not allow for calculation of an MDR and resulted in exclusion of the study from the database. Understanding changes in effect has limited utility because risk assessments are conducted on the basis of effective concentrations. In order to test IA in a way that MDRs can be calculated, the method design must allow predicted and observed effective concentrations, such as median lethal concentration, to be calculated. Examples of this approach are contained in Belden and Lydy (2006), Cedergreen and Striebig (2005), and Faust et al. (2000).

The study designs and quality control varied among the experiments included in the compiled database. All studies were presented in peer-reviewed publications. However, because the current study did not include evaluations of measured versus nominal concentration or on other standard quality-control protocols, users of the data should refer to the original references for further details. An additional factor that was not evaluated was whether or not individual toxicity tests were conducted concurrently within each mixture study. If tests were not conducted concurrently, shifts in health of the test organisms or other factors may influence the outcome of the study. Few studies that were reviewed directly stated that tests were conducted concurrently.

The reviewed experiments tested many different pesticides and a variety of test organisms. One hundred twenty seven active pesticide ingredients were tested (listed in Supplemental Table 6). Several pesticides have been tested extensively. For example, atrazine was a component of a mixture in $17 \%$ of the experiments (50 of 303) and chlorpyrifos was a component in $15 \%$ (44 of 303). However, several combinations that are frequent contaminants in surface water have not been tested at all. For example, a recent study investigating pesticide occurrence in streams near corn/soybean cropping systems found that triazine and acetanilide herbicides form combinations that are of the greatest toxicological concern in regard to green algae and duckweed endpoints (Belden et al. 2007). Despite the predictability of their co-occurrence due to applications in the same crop settings, combinations of these groups have not been studied extensively. Prioritization of pesticide mixtures using systematic strategies based on occurrence and potential toxicity can be used to identify needs and priorities for future testing (Belden et al. 2007).

Several higher taxonomic groups, including fish, amphibians, bivalves, insects, crustaceans, green algae, and duckweed, have been used for mixture toxicity tests (Table 1); however, a few species have dominated testing. For example, Scenedesmus vacuolatus (green algae), Lemna minor (duckweed), and Chironomus tentans (aquatic invertebrate) accounted for $27 \%, 19 \%$, and $18 \%$ of experiments, respectively 
Table 1. The number of experiments testing concentration addition (CA), independent action (IA), and simple interaction (SI) models for each taxonomic group. $n=303$

\begin{tabular}{|l|r|r|r|}
\hline & \multicolumn{3}{|c|}{ Predictive model } \\
\hline Higher taxonomic group & CA & IA & SI \\
\hline Amphibia & 4 & - & 3 \\
\hline Bivalvia & 2 & - & - \\
\hline Chlorophyta & 87 & 10 & - \\
\hline Crustacea & 20 & 4 & 14 \\
\hline Insecta & 16 & 1 & 39 \\
\hline Liliopsida & 38 & 19 & - \\
\hline Osteichthyes & 38 & 1 & 3 \\
\hline
\end{tabular}

(Supplemental Table 7). L. minor was the only vascular plant tested. Overall for CA experiments, $77 \%$ of experiments were conducted with 1 of 5 species and $90 \%$ of experiments were conducted with 1 of 10 species. For IA, other than 2 community level tests (photosynthesis inhibition as endpoint), all other tests were conducted on 7 species, with the majority of experiments utilizing green algae and duckweed (37 total tests, Supplemental Table 7). In SI experiments, nearly all work has been based on triazine herbicide potentiation of insecticides. Additionally, nearly all of these studies have been conducted on 1 of 2 organisms - the insect Chironomus tentan and the crustacean Hyalella azteca (Supplemental Table 7).

Endpoints used in most of these studies were acute in nature, with mobility or lethality evaluated for animals and simple short-term growth measurements evaluated for plants. Generally, only these few commonly measured endpoints were evaluated for most studies. Although experimental evidence with duckweed has suggested that selection of specific endpoints can greatly influence the results of mixtures studies (Cedergreen and Siebig 2005), resource limitations usually limit mixture studies. For example, most mixture experiments involve evaluation of at least 50 experimental units; thus, it often is not deemed feasible to run long-term studies. In addition, nearly all tests have been performed using single species and only a few studies have attempted to use CA models to predict toxic response at a community level (Vighi et al. 2003; Arrhenius et al. 2004). Further testing is needed to evaluate predictive models using chronic and multiple species designs.

\section{Assessment of model accuracy}

Several methods have been developed to express the deviation of observed toxicity from the toxicity predicted by models. Generally, approaches either use a linear scale that allows for even distribution of values above and below predicted or they use a simple ratio. In this study, emphasis has been placed on a simple ratio, the MDR, for expressing deviation throughout the dataset. MDR is defined as

$$
M D R=\frac{\text { Expected }}{\text { Observed }}
$$

where Expected is the effective concentration of the mixture that would be predicted by the model and Observed is the effective concentration for the mixture obtained from toxicity testing (Belden and Lydy 2006). In the current study, the effective concentration to $50 \%$ of the population (EC50) or lethal concentration to $50 \%$ of the population (LC50) were used as the effective concentration. The MDR values were chosen as the reference scale because of the simplicity of adjusting values from predictive models. Risk assessors can simply divide the level of potential toxicity that is predicted by a mixture model by the MDR to adjust empirical data for the mixture of interest. If a linear scale is needed for better visualization of model fit, MDR values can be plotted on a log scale to achieve linearity. Similarly, in order to facilitate discussion, MDR ranges also are expressed as factors of the predicted values. Thus, observed values that fall within a factor of 2 of the predicted value $(2 \times)$ are those with MDR values between 0.5 and 2.0.

The MDR values were obtained from the experiments reviewed for this study via the following approaches, depending on the information reported for each experiment.

Approach 1: Used as reported in the experimental results as $M D R$, interactivity ratio, or synergistic ratio-Interactivity ratios (Denton et al. 2003) and synergistic ratios (Belden and Lydy 2000) are equivalent to the MDR and the numbers are used directly as are reported MDR values (Belden and Lydy 2006).

Approach 2: Computed from observed and predicted valuesIf experiments provide the expected and observed effective concentrations, then MDR values can be calculated using Equation 4 (Altenburger et al. 2000).

Approach 3: Computed from TU values-Observed TU values have been calculated by determining the TU value at which 50\% effect occurred during experimentation (Eqn. 3; Pape-Lindstrom and Lydy 1997). The expected value would be 1 TU. The reciprocal of the TU value is equivalent to MDR.

Approach 4: Computed from the additive index reported for the experiment-The additive index ([AI]; Marking and Mauck 1975 ) is similar to the TU in the initial calculation: $A_{m} / A_{i}+$ $B_{m} / B_{i}=S$, where $A$ and $B$ are LC50 values for toxicants in the mixture $(m)$ and individually $(I)$. Thus, $S$ is basically a toxic unit from Approach 3. However, the additive index further transforms $S$ as follows:

If $S \leq 1$, then the $\mathrm{AI}=1 / S-1.0$

If $S \geq 1$, then the $\mathrm{AI}=S(-1)+1$

Thus, if the $\mathrm{AI}$ is negative,

$$
\mathrm{MDR}=1 /(\mathrm{AI}-1)(-1)
$$

If the $\mathrm{AI}$ is positive,

$$
\mathrm{MDR}=\mathrm{AI}+1
$$

Approach 5: Computed from the isobologram approach or other approaches that provide LC50 for each toxicant and for a mixture of known ratio-The ratio of toxicants in the mixture is used to divide the LC50 of the mixture into individual concentrations for each component. The individual concentrations then can be transformed into TUs based on the individual LC50 values. Approach 3 then can be used to calculate the MDR. Example experiments include NorbergKing et al. (1991) and Cedergreen and Streibig (2005).

For some studies, the above information was not available and MDR values could not be calculated. These studies were not used in calculations presented within the study. 


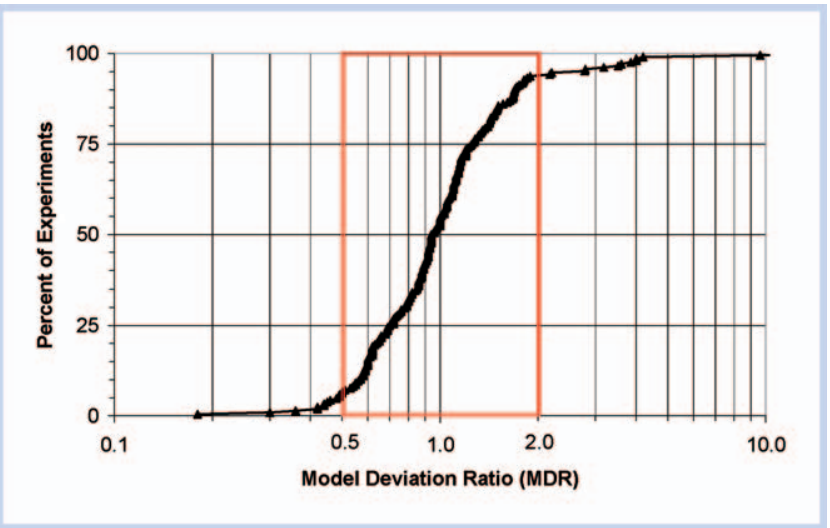

Figure 1. The cumulative distribution of model deviation ratios (MDR) for all 207 concentration addition experiments. An MDR of 1.0 indicates perfect fit to the model. Greater than 1 indicates greater toxicity than expected, and less than 1 indicates less toxicity than expected. The double-lined box delineates values that are within a factor of 2 from the predictive model. Each triangle indicates an MDR value.

\section{RESULTS: ANALYSIS OF MODEL PREDICTIONS}

\section{$C A$ and IA}

Model deviation ratios for CA experiments are summarized in Figures 1 and 2 and provided individually in the Supplemental Tables. In all Figures, the MDR range from 0.5 to 2.0 is indicated. MDR values within this range indicate that observed values were within a factor of 2 of predicted values. Although arbitrary, the range provides a benchmark for discussing the accuracy of the models. Considering all experiments evaluating the CA model, regardless of MOA and pesticide target groups, $88 \%$ had observed effective concentrations within the MDR range of 0.5 and 2.0. The median MDR was near 1 , only about $5 \%$ of MDRs were less than 0.5 , and about $5 \%$ were greater than 2 , indicating unbiased estimates overall (Figure 1).

After sorting experiments based on similar MOA and pesticide use group (Figure 2), the best overall agreement between CA predictions and observed toxicity, as expected from results of previous studies, was for pesticides with the same MOA (highest proportion of MDRs close to 1.0). Similar to the combined results in Figure 1, more than $85 \%$ of these same-MOA experiments had MDR values between 0.5 and 2.0. However, there was a general tendency for the actual toxicity of the same-MOA mixtures to be greater than predicted by CA, with about $70 \%$ of the experiments having MDR values of greater than 1, and about $10 \%$ of experiments having MDR values of greater than 2 (Figure 2). In groups with different MOAs, including those in the same pesticide group and those from different pesticide groups, the MDR distributions were relatively similar. The CA model had more of a tendency to overpredict toxicity for these 2 differentMOA groups (compared to the same MOA group), with $66 \%$ and $47 \%$ rates of overprediction, respectively. Although the majority of experiments within either group had MDR values within a range of 0.5 to 2.0 , the values tended to be more variable than found for experiments with compounds of the same MOA (Figure 2).

All experiments that tested the IA model used methodologies that allowed calculation of the CA model as well (Altenburger et al. 2003 for methods; Belden and Lydy 2006). Through analysis of this subset of experiments, direct

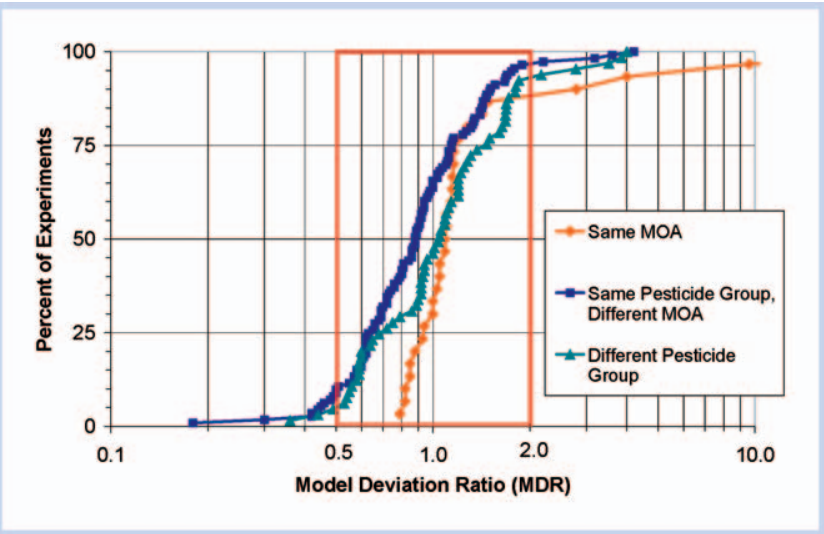

Figure 2. The cumulative distribution of model deviation ratios (MDR) for concentration addition experiments grouped by mixtures with the same mode of action ([MOA]; $n=30$, indicated by diamond symbol), mixtures of the same pesticide use group and different MOA $(n=113$, indicated by square symbol), and mixtures of different pesticide use groups and different MOA ( $n=64$, indicated by square symbol). The double-lined box delineates values that are within a factor of 2 from the predictive model. An MDR of 1.0 indicates perfect fit to the model. Greater than 1 indicates greater toxicity than expected and less than 1 indicates less toxicity than expected.

comparisons of the methods based on MDR distributions were made and are shown for same-MOA pesticides, different MOA but same group, and different MOA and different group (Figure 3). In each MOA and pesticide group, IA tended to somewhat overpredict toxic concentrations, thus underestimating toxicity compared to the CA model. This difference was greatest for mixtures with the same MOA for which CA predictions resulted in MDR values from 0.75 to 2.0, although IA resulted in MDRs in exceedance of 2.0 in 3 of 7 experiments (Figure 3). Conversely, for mixtures having different MOAs, but that were from the same pesticide group, the 2 models were very similar in prediction results (Figure 3). Both models tended to overpredict toxicity, with greater than $70 \%$ of MDR values less than 1.0. Finally, for mixtures from different pesticide groups, the models also had relatively similar distributions of MDRs, with nearly all experiments having MDR values within a factor of 2 of predicted toxicity. However, IA was somewhat more accurate, with a near equal distribution of values above and below predicted values, whereas CA tended to overpredict toxicity (Figure 3).

Separations of pesticides based on MOA are complicated due to overlapping physiology effects among pesticide classes and secondary modes of actions for many pesticides. The relationship between related, but different, MOAs and predictability of toxicity by IA and CA has not been well tested. It should be noted that, in this study, only pesticides within the same chemical class and that had the same target receptor site were classified as having the same MOA. This division is the least subjective split because chemical classes of pesticides generally are less disputed than the extent to overlapping modes of action among products.

\section{Multicomponent mixtures}

Mixture studies can be conducted using either simple binary or tertiary mixtures, or more complex, multiplecomponent mixtures (Supplemental Tables 1-3). Previous investigators have suggested that a funneling effect tends to make the CA model a reasonable approximation for complex mixtures (Warne and Hawker 1995; Deneer 2000). In other words, although some specific mixtures may not fit the CA 


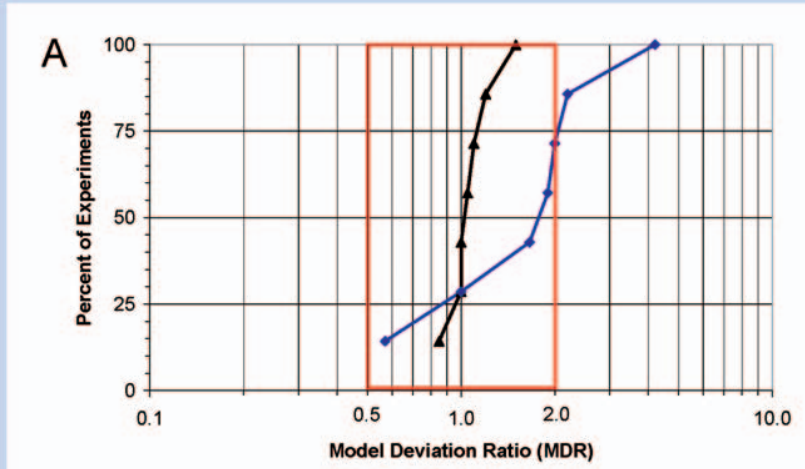

B
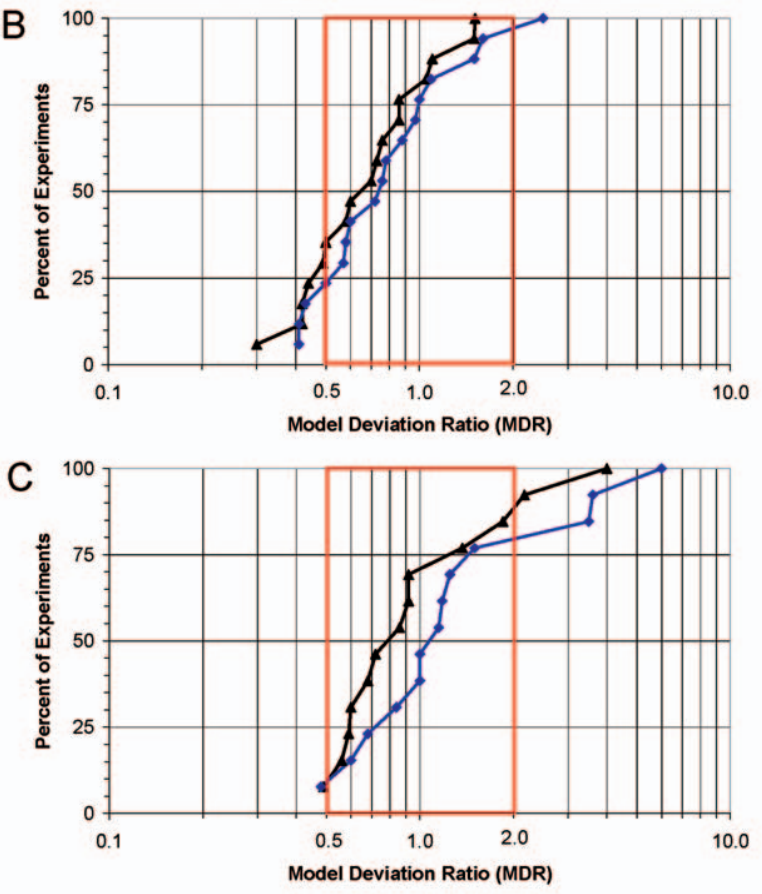

Figure 3. The cumulative distribution of model deviation ratios (MDR) that evaluated both independent action ([IA], indicated by diamonds) and concentration addition ([CA], indicated by triangles) models for pesticide mixtures that $(\mathrm{A})$ contained pesticides with the same mode of action $(n=7)$; (B) contained pesticides with different modes of action, but the same pesticide group (17); and (C) contained pesticides from different pesticide groups $(n=13)$. An MDR of 1.0 indicates perfect fit to the model and the double-lined box delineates values that are within a factor of 2 from the predictive model. Greater than 1 indicates greater toxicity than expected and less than 1 indicates less toxicity than expected.

model, when they occur in a multicomponent mixture these nonadditive combinations will have a negligible effect when combined with other pesticide mixtures that are mostly additive. Although this theory is not well tested, results from this study suggest that the theory has merit. All 11 studies that tested complex multicomponent mixtures (4 or more components) had MDR values from 0.75 to 2.0, and 9 of these studies had MDR values that closely matched the CA model with MDR values from 0.75 to 1.5 . Although the dataset is small, this suggests better predictions by CA for these complex mixtures than would be expected based on the entire range of mixtures included in the complete dataset (Figure 1). However, care should be taken not to select models and evaluate uncertainties based solely on the multicomponent studies. Studies evaluating field exposures often have indicated that most toxicity likely is due to a few

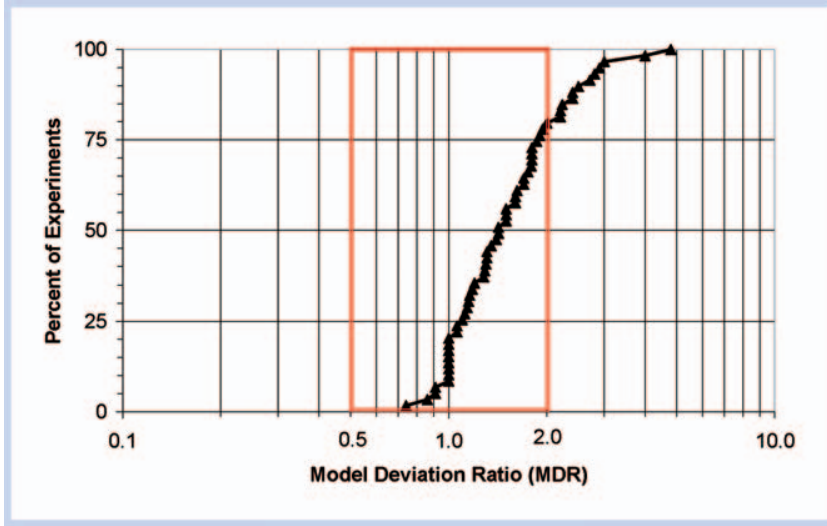

Figure 4. The cumulative distribution of model deviation ratios (MDR) for concentration addition (CA) model estimates for all simple interaction (SI) experiments $(n=59)$. An MDR of 1.0 indicates perfect fit to the CA model. The double-lined box delineates values that are within a factor of 2 from the predictive model. Greater than 1 indicates greater toxicity than expected and less than 1 indicates less toxicity than expected.

components within a mixture that are present at high concentrations relative to their effective concentrations (Norberg-King et al. 1991; Amweg et al. 2006; Belden et al. 2007). Therefore, the uncertainties associated with predicting the toxicity of simple mixtures likely is more realistic for applications to most environmental exposures. Binary toxicity tests and studies evaluating environmental mixtures (Olmstead and LeBlanc 2005; Junghans et al. 2006) are also important areas of continued testing.

\section{Interactions}

Deviations of model predictions from observations of actual toxicity can be due to either selection of the wrong model or because an interaction has occurred. Currently, we are unable to predict if an interaction will occur for most mixtures. The experiments reviewed for this study indicate that interactions do not routinely result in large differences in toxicity from the CA model, because the majority of MDR values fell less than 2.0 (Figures 1 and 2).

Of specific concern is the potential that a contaminant present at concentrations below its expected effect level (as an individual contaminant or its contribution to a mixture through CA or IA) is a synergist such that it acts to increase the toxicity of the combination of contaminants. Currently, risk assessments conducted a priori are not capable of dealing with this scenario. Thus, studies evaluating this possibility through the SI approach are of interest. Figure 4 shows the distribution of MDR values for experiments evaluating SI. Nearly all experiments demonstrated MDR values of 1.0 or greater, and more than 20\% of experiments indicated MDR values greater than 2.0. Taken at face value, this result would raise a concern that mixtures of contaminants, although individually at low concentrations, frequently may increase the toxicity of the mixture as a whole. However, this dataset is biased due to a large number of studies evaluating whether atrazine and other triazine herbicides may potentiate organophosphate insecticides based on initial work by PapeLindstrom and Lydy (1997; Supplemental Table 5). Other studies in the dataset also were conducted based on previous indications that specific combinations may result in an interaction. Due to inadequate data and toxicological knowledge, it is not currently possible to estimate how frequently 
Table 2. Percentile values for model deviation ratios (MDR) for each group of concentration addition experiments. MOA = mode of action

\begin{tabular}{|l|c|c|c|c|}
\hline & \multicolumn{5}{|c|}{ Percentile } \\
\hline Group & 80 & 90 & 95 & 99 \\
\hline All & 1.40 & 1.69 & 2.58 & 4.19 \\
\hline Same MOA & 1.31 & 2.91 & 7.08 & 104 \\
\hline Same pesticide use group, different MOA & 1.32 & 1.51 & 1.77 & 3.55 \\
\hline Different pesticide use group & 1.65 & 1.81 & 2.66 & 3.90 \\
\hline
\end{tabular}

this type of interaction occurs from pesticide mixtures that commonly occur rather than in mixtures that were specifically chosen based on potential for an interaction to occur.

\section{CURRENT APPLICABILITY OF MIXTURE MODELS AND MDR TO RISK ASSESSMENT PRACTICES}

\section{Model selection}

The lst consideration prior to incorporation of mixture toxicity into risk assessments is model selection. The subset of data comparing the CA and IA models, presented in Supplemental Tables 3 through 5, generally support the conclusion of several studies that suggest that mixtures of contaminants with the same MOA tend to be best modeled by $\mathrm{CA}$ and the mixtures with different MOA tend to be best modeled by IA (Faust et al. 2000; Altenburger 2003; Backhaus et al. 2004). This finding was especially significant for mixtures with the same MOA, where CA accurately predicted toxicity and IA underpredicted toxicity (Figure 3). However, the differences between the models for experiments with different MOAs were not great, and model selection may be governed more appropriately by the goal of selecting the more conservative of the models; the CA model has the overall tendency to provide conservatively high estimates of toxicity compared to the IA model, yet with quite similar overall predictive accuracy. It also should be noted that this subset summarizes only 37 experiments (Figure 3).

In contrast to the small dataset available for testing both models, a much larger dataset (207 experiments) is available for CA (Figures 1 and 2). In these experiments, the CA model predictions are relatively accurate and unbiased relative to observed toxicity. Furthermore, CA tends to be the more conservative model compared to IA (Figure 3), as has been theorized previously to occur when dose-response slopes are steep (Drescher and Boedeker 1995), which is likely for most aquatic pesticide exposures. Because of the larger dataset, the generally conservative nature of $\mathrm{CA}$, and the greater ease of use, this model is probably the best choice for most riskassessment applications at this time, especially when the emphasis of the assessment is based on conservatively indicating potential risks to the environment, such as in a proactive or screening-level risk assessment.

Although no previous reviews have been conducted using IA or SI, 2 other reviews have evaluated the accuracy of CA (Deneer 2000; Lydy et al. 2004). In both of these studies, deviation from models generally was considered to be $3 \times$ or less, thus generally matching the current study. However, Deneer (2000) did not fully describe how deviation from CA was determined and, because it was published in 2000, does not include results from recent experiments. Lydy et al.
(2004) focused more on the mechanisms of mixture risk assessment and provided only a qualitative assessment of deviations from the CA model.

\section{Use of empirical data}

Direct observations from laboratory experiments with pesticide mixtures are necessary to completely understand the potential joint action and interactions for specific mixtures that previously have not been studied. Although numerous toxicity studies have been performed with pesticide mixtures, as reviewed by this study, the amount of data available is small compared to the large number of mixtures that occur in the environment. In order to enhance our understanding of the interactions between and among individual pesticides and combinations of pesticides, more studies of mixture toxicities are needed and these studies should assess the mixtures of greatest potential importance (occurrence and toxicity) in aquatic ecosystems (Belden et al. 2007), including the testing of more species and acute and chronic endpoints. The dataset provided in the Supplemental Material section provides the start to obtaining a uniform source of empirically derived uncertainties for specific mixtures.

Empirical data also can be used to help us understand the types of compounds that may interact and thus may be important to consider even if the individual compounds are present at concentrations well below toxic levels. For example, numerous studies have found interactions among organophosphate insecticides and triazine herbicides (reviewed by Lydy et al. 2004), thus indicating that mixtures of these pesticides may warrant a higher degree of scrutiny.

\section{Accounting for uncertainty}

The frequency distributions of the MDR values compiled in this study provide a means to account for uncertainty in estimates from predictive models. For example, Table 2 lists MDR values for several specific percentiles from the datasets based on the CA model (Figures 1 and 2). These values can be used to determine safety factors to account for potential deviations from mixture models and limit the potential for error to a particular probability. For example, if the goal is to limit the risk of underestimating toxicity with the CA model to $10 \%$, then the 90 th percentile MDR of 1.69 (assuming mixed MOAs and pesticide groups) can be multiplied by the CA estimate of toxicity (or divided into the estimated toxicity-normalized total concentration of the mixture).

Note that the percentiles listed in Table 2 only account for uncertainty from mixture models and not for mixtures entirely. For example, Belden et al. (2007) noted that the summed TU value of mixtures was generally not higher than a 
factor of 2 above the highest toxicity quotient (highest individual TU) for green algae and duckweed in corn-soybean row crop systems under nonstorm event conditions. However, the current paper indicates that a deviation of 2.61 may occur from CA (all data, 95\%; Table 2); thus, the overall uncertainty related to the presence of mixtures as compared to only the most toxic component would be greater than a factor of 5 for this pesticide-usage landscape in low-flow conditions if toxicity is attributed only to the toxicant present at the highest individual TU. It would be expected that other pesticide-usage landscapes that utilize a greater variety of active ingredients might have larger uncertainty factors.

\section{Future research needs}

Assessment of the effects of pesticide mixtures on aquatic ecosystems is still in developmental stages. Additional studies are needed to further develop predictive models, such as combined IA/CA approaches (Altenburger et al. 2004; Olmstead and LeBlanc 2005) and to evaluate the performance of mixture models for predicting mixture toxicity when evaluating community endpoints, long-term exposure, and no-observed-effect levels. Furthermore, approaches for predicting the occurrence or likelihood of interactions need to be developed. Approximately $10 \%$ of all studies that were evaluated had MDRs greater than 2.0 for the CA model, indicating the potential of synergistic interactions. If there are specific combinations of pesticides that account for these most extreme deviations, then these specific combinations are important to further examine with regard to their toxicity and their frequency of occurrence in the environment. As modeling and mechanistic refinements are being developed, concurrent efforts are warranted to further develop riskassessment procedures based on our current knowledge of the composition of pesticide mixtures that occur in the environment and by applying available models using approaches that account for model bias and uncertainty.

Acknowledgment-This work was partially funded by the National Water Quality Assessment Program, US Geological Survey.

\section{SUPPORTING INFORMATION}

Table S1. Experiments conducted testing a concentration addition model using pesticides with the same mode of action. The model deviation ratio (MDR) is calculated by dividing the expected median effective concentration by the measured median effective concentration of the mixture. Refer to text for MDR Methods. Deviation from the model is based on reporting from the original paper.

Table S2. Experiments conducted testing a concentration addition model using pesticides from the same class but with differing modes of action. The model deviation ratio (MDR) is calculated by dividing the expected median effective concentration by the measured median effective concentration of the mixture. Refer to text for MDR Methods. Deviation from the model is based on reporting from the original paper.

Table S3. Experiments conducted testing a concentration addition model using pesticides from different classes. The model deviation ratio (MDR) is calculated by dividing the expected median effective concentration by the measured median effective concentration of the mixture. Refer to text for MDR Methods. Deviation from the model is based on reporting from the original paper.

Table S4. Experiments conducted testing independent action models. The model deviation ratio (MDR) is calculated by dividing the expected median effective concentration by the measured median effective concentration of the mixture. Refer to text for MDR Methods. Deviation from the model is based on reporting from the original paper.

Table S5. Experiments conducted testing if interactions are occurring. The model deviation ratio (MDR) is calculated by dividing the expected median effective concentration by the measured median effective concentration of the mixture. The expected model is the concentration response curve of the toxicant applied at known toxic level. The potential "synergist" or interactive agent is shown in bold and was only present at concentrations below that expected to cause toxicity by either the CA or IA models.

Tables S1-S5 found at DOI: 10.1897/2006-046.S1 (86 KB PDF)

Table S6. Alphabetical list of pesticides that were evaluated in mixture toxicity tests. The number of studies that used each pesticide is shown based on experimental design (CA = concentration addition; IA = independent action; $\mathrm{SI}=$ simple interaction). The CA studies also are divided by pesticide mode of actions (MOA) and classes as described in the paper.

Table S7. Alphabetical list of species that were evaluated in mixture toxicity tests. The number of studies that used each species is shown based on experimental design $(\mathrm{CA}=$ concentration addition; IA = independent action; $\mathrm{SI}=$ simple interaction). The CA studies also are divided by pesticide mode of actions (MOA) and classes as described in the paper.

Tables S6 and S7 found at DOI: 10.1897/2006-046.S2 (pages el-e5, web-only html)

\section{REFERENCES}

Altenburger R, Backhaus T, Boedeker W, Faust M, Scholze M, Grimme LH. 2000. Predictability of the toxicity of multiple chemical mixtures to Vibrio fischeri: Mixtures composed of similarly acting chemicals. Environ Toxicol Chem 19:2341-2347

Altenburger R, Nendza M, Schuurmann G. 2003. Mixture toxicity and its modeling by quantitative structure-activity relationships. Environ Toxicol Chem 22:1900-1915

Altenburger R, Walter H, Grote M. 2004. What contributes to the combined effect of a complex mixture? Environ Sci Technol 38:6353-6362.

Amweg EL, Weston DP, You J, Lydy MJ. 2006. Pyrethroid insecticides and sediment toxicity in urban creeks from California and Tennessee. Environ Sci Technol 40:1700-1706

Arrhenius A, Gronvall F, Scholze M, Backhaus T, Blanck H. 2004. Predictability of the mixture toxicity of 12 similarly acting congeneric inhibitors of photosystem II in marine periphyton and epipsammon communities. Aquatic Toxicol 68:351-367.

Backhaus T, Altenburger R, Boedeker W, Faust M, Scholze M, Grimme LH. 2000 Predictability of the toxicity of a multiple mixture of dissimilarly acting chemicals to Vibrio fischeri. Environ Toxicol Chem 19:2348-2356.

Backhaus T, Faust M, Scholze M, Gramatica P, Vighi M, Grimme LH. 2004. Joint algal toxicity of phenylurea herbicides is equally predictable by concentration addition and independent action. Environ Toxicol Chem 23:258-264.

Belden JB, Gilliom RJ, Martin JD, Lydy MJ. 2007. Relative toxicity and occurrence patterns of pesticide mixtures in streams draining agricultural watersheds dominated by corn and soybean production. Integ Environ Assess Manag 3:90-100.

Belden JB, Lydy MJ. 2000. Impact of atrazine on organophosphate insecticide toxicity. Environ Toxicol Chem 19:2266-2274.

Belden JB, Lydy MJ. 2006. Joint toxicity of chlorpyrifos and esfenvalerate to fathead minnows and midge larvae. Environ Toxicol Chem 25:623-629. 
Boone MD, James SM. 2003. Interactions of an insecticide, herbicide, and natural stressors in amphibian community mesocosms. Ecol Appl 13:829-841.

Carder JP, Hoagland KD. 1998. Combined effects of alachlor and atrazine on benthic algal communities in artificial streams. Environ Toxicol Chem 17:1415-1420.

Cedergreen N, Kamper A, Streibig JC. 2006. Is prochloraz a potent synergist across aquatic species? A study on bacteria, daphnia, algae, and higher plants. Aquat Toxicol 78:243-252.

Cedergreen N, Streibig JC. 2005. Can the choice of endpoint lead to contradictory results of mixture-toxicity experiments? Environ Toxicol Chem 24:1676-1683.

Chevre N, Loeppe C, Singer H, Stamm C, Fenner K, Escher BI. 2006. Including mixtures in the determination of water quality criteria for herbicides in surface water. Environ Sci Technol 40:426-435.

Cuppen JGM, Crum SJH, Van den Heuvel HH, Smidt RA, Van den Brink PJ. 2002. Effects of a mixture of two insecticides in freshwater microcosms: I. Fate of chlorpyrifos and lindane and responses of macroinvertebrates. Ecotoxicology 11:165-180.

Deneer JW. 2000. Toxicity of mixtures of pesticides in aquatic systems. Pest Manag Sci 56:516-520

Denton DL, Wheelock CE, Murray SA, Deanovic LA, Hammock BD, Hinton DE. 2003. Joint acute toxicity of esfenvalerate and diazinon to larval fathead minnows (Pimephales promelas). Environ Toxicol Chem 22:336-341.

Drescher K, Boedeker W. 1995. Assessment of the combined effects of substances: The relationship between concentration addition and independent action. Biometrics 51:716-730.

Fairchild JF, Lapoint TW, Schwartz TR. 1994. Effects of an herbicide and insecticide mixture in aquatic mesocosms. Arch Environ Contam Toxicol 27:527-533.

Faust M, Altenburger R, Backhaus T, Bodeker W, Scholze M, Grimme LH. 2000. Predictive assessment of the aquatic toxicity of multiple chemical mixtures. J Environ Qual 29:1063-1068.

Faust M, Altenburger R, Backhaus T, Blanck H, Boedeker W, Gramatica P, Hamer V, Scholze M, Vighi M, Grimme LH. 2001. Predicting the joint algal toxicity of multicomponent s-triazine mixtures at low-effect concentrations of individual toxicants. Aquatic Toxicol 56:13-32.

Faust M, Altenburger R, Boedeker W, Grimme LH. 1993. Additive effects of herbicide combinations on aquatic nontarget organisms. Sci Total Environ Supplement: 941-951.

Gilliom RJ, Barbash JE, Crawford CG, Hamilton PA, Martin JD, Nakagaki N, Nowell LH, Scott JC, Stackelberg PE, Thelin GP, Wolock DM. 2006. The quality of our nation's waters - pesticides in the nation's streams and groundwater, 19922001. Reston (VA): US Geological Survey. Circular 1291.

Gilliom RJ, Barbash JE, Kolpin DW, Larson SJ. 1999. Testing water quality for pesticide pollution. Environ Sci Technol 33:16A-169A.
Hayes TB, Case P, Chui S, Chung D, Haeffele C, Haston K, Lee M, Mai VP, Marjuoa Y, Tsui M. 2006. Pesticide mixtures, endocrine disruption, and amphibian declines: Are we understanding the impact? Environ Health Perspect 114:40-50.

Hewlett PS. 1969. Measurement of the potency of drug mixtures. Biometrics 25:477-487.

Hoagland KD, Drenner RW, Smith JD, Cross DR. 1993. Freshwater community responses to mixtures of agricultural pesticides - effects of atrazine and bifenthrin. Environ Toxicol Chem 12:627-637.

Junghans M, Backhaus T, Faust M, Scholze M, Grimme LH. 2003. Predictability of combined effects of eight chloroacetanilide herbicides on algal reproduction. Pest Manag Sci 59:1101-1110.

Junghans M, Backhaus T, Faust M, Scholze M, Grimme LH. 2006. Application and validation of approaches for the predictive hazard assessment of realistic pesticide mixtures. Aquat Toxicol 76:93-110.

Lydy MJ, Austin KR. 2005. Toxicity assessment of pesticide mixtures typical of the Sacramento-San Joaquin Delta using Chironomus tentans. Arch Environ Contam Toxicol 48:49-55.

Lydy M, Belden J, Wheelock C, Hammock B, Denton D. 2004. Challenges in regulating pesticide mixtures. www.ecologyandsociety.org/vol9/iss6/art1. Accessed 12 December 2005.

Macek KJ. 1975. Acute toxicity of pesticide mixtures to bluegills. Bull Environ Contam Toxicol 14:648-652.

Marking LL, Mauck WL. 1975. Toxicity of paired mixtures of candidate forest insecticides to rainbow trout. Bull Environ Contam Toxciol 13:518-523.

Norberg-King TJ, Durhan EJ, Ankley GT. 1991. Application of toxicity identification evaluation procedures to the ambient waters of the Colus Basin Drain California. Environ Toxicol Chem 10:891-900.

Olmstead AW, LeBlanc GA. 2005. Toxicity assessment of environmentally relevant pollutant mixtures using a heuristic model. Integ Environ Assess Manag 1:114-122.

Pape-Lindstrom PA, Lydy MJ. 1997. Synergistic toxicity of atrazine and organophosphate insecticides contravenes the response addition mixture model. Environ Toxicol Chem 16:2415-2420.

[USEPA] US Environmental Protection Agency. 2000. Supplementary guidance for conducting health risk assessment of chemical mixtures. Washington DC: USEPA. EPA/630/R-00/002.

Vighi M, Altenburger R, Arrhenius A, Backhaus T, Bodeker W, Blanck H, Consolaro F, Faust M, Finizio A, Froehner K, Gramatica P, Grimme LH, Gronvall F, Hamer V, Scholze M, Walter H. 2003. Water quality objectives for mixtures of toxic chemicals: Problems and perspectives. Ecotoxicol Environ Saf 54:139-150.

Warne MSJ, Hawker DW. 1995. The number of components in a mixture determines whether synergistic and antagonistic or additive toxicity predominate: The funnel hypothesis. Ecotoxicol Environ Saf 31:23-28. 